\title{
Race/ethnicity, substance abuse, and mental illness among suicide victims in 13 US states: 2004 data from the National Violent Death Reporting System
}

\author{
D L Karch, L Barker, T W Strine
}

Injury Prevention 2006;12(Suppl II):ii22-ii27. doi: 10.1136/ip.2006.013557

See end of article for authors' affiliations

Correspondence to: Dr D Karch, Centers for Disease Control and Prevention, National Center for Injury

Prevention and Control, 4770 Buford Highway, $\mathrm{NE}$, Mailstop K-60,

Atlanta, GA 30341, USA

DKarch@cdc.gov

Accepted 30 August 2006

\begin{abstract}
Objective: To calculate the prevalence of substance abuse and mental illness among suicide victims of different racial/ethnic groups and to identify race/ethnicity trends in mental health and substance abuse that may be used to improve suicide prevention.

Methods: Data are from the National Violent Death Reporting System (NVDRS), a state-based data integration system that, for 2004, includes data from 13 US states. The NVDRS integrates medical examiner, toxicology, death certificate, and law enforcement data.

Results: Within participating states, for data year 2004, 6865 suicide incidents in which race/ethnicity are known were identified. This included 5797 (84.4\%) non-Hispanic whites, 501 (7.3\%) non-Hispanic blacks, 257 (3.7\%) Hispanics, and 310 (4.5\%) persons from other racial/ethnic groups. At the time of the suicide event, non-Hispanic blacks had lower blood alcohol contents than other groups. Non-Hispanic whites had less cocaine but more antidepressants and opiates. There were no differences in the levels of amphetamines or marijuana by race/ethnicity. Hispanics were less likely to have been diagnosed with a mental illness or to have received treatment, although family reports of depression were comparable to non-Hispanic whites and other racial/ethnic groups. Non-Hispanic whites were more likely to be diagnosed with depression or bipolar disorder and non-Hispanic blacks with schizophrenia. Comorbid substance abuse and mental health problems were more likely among non-Hispanic whites and nonHispanic blacks, while Hispanics were more likely to have a substance abuse problem without comorbid mental health problems.

Conclusion: The results support earlier research documenting differences in race/ethnicity, substance abuse, and mental health problems as they relate to completed suicide. The data suggest that suicide prevention efforts must address not only substance abuse and mental health problems in general, but the unique personal, family, and social characteristics of different racial/ethnic groups.
\end{abstract}

$T^{\prime}$ here were over 31000 suicides in the US in 2003. Age adjusted suicide rates in 2003 for non-Hispanic whites were 12.6 per 100 000, 5.4 for non-Hispanic blacks, 5.6 for Hispanics, and 6.6 for other races/ethnicities. ${ }^{1}$ Suicide was the second leading cause of death for all persons aged 25-34 years, the third leading cause for persons aged 10-14 years, the fourth leading cause for persons aged 35-44, and the fifth leading cause for persons age $45-54$ years. ${ }^{1}$ Thus, suicide was among the top five causes of death for all people in the US aged $10-54$ years.

Only minor variations in leading causes of death are noted when looking at suicide rates by race. Suicide is the second, third, and fourth leading cause of death for non-Hispanic whites aged 15-34 years, 10-14 years, and 35-54 years respectively. For non-Hispanic blacks, suicide is the third leading cause of death for persons 15-24 years and the fifth leading cause for persons aged 10-14 years. Suicide for Hispanics is the third leading cause of death for persons age 10-24 and the fourth leading cause of death for persons aged 25-34 years.

Research in the area of race/ethnicity, substance abuse, and mental illness, as they contribute to suicide, has typically been done from the perspective of living people expressing suicidal ideation or surviving a suicide attempt. ${ }^{2-7}$ Often these studies rely on self-report data from patients currently receiving care for suicidal ideation, substance abuse, or mental illness, and attempt to identify risk and protective factors so that early intervention techniques can be applied.
Fewer studies have looked at these factors from the perspective of a psychological autopsy (intensive interviews with surviving relatives and friends) combined with coroner/ medical examiner and law enforcement professionals involved in investigating completed suicides. ${ }^{8}$

Studies that focus on completed suicides indicate a high use of alcohol and drugs at the time of death. Medical examiner records indicate that the percent of decedents with alcohol in their system at the time of suicide has been found to be as high as $38.8 \%$ to $69 \% .^{9}{ }^{10}$ One study found $45 \%$ of suicide cases were positive for alcohol with 19\% having a blood alcohol concentration (BAC) greater than $0.150 \mathrm{~g} / \mathrm{dl}$ or almost twice the legal intoxication limit in most states. ${ }^{11}$ Other studies highlight significant differences in pre-suicide alcohol intoxication and drug intoxication by different racial/ ethnic groups. ${ }^{10-12}$ Studies of completed suicides also indicate a high prevalence of mental illness, especially depression, and an even greater risk when mental illness is combined with substance abuse. ${ }^{16-19}$ As with alcohol and drugs alone, studies have also found significant differences in risk among racial/ethnic groups as it pertains to the combination of mental illness and substance abuse. ${ }^{13} 19$

Many of the studies that focus on completed suicide and the impact of race/ethnicity, mental illness, or substance abuse collect their data in a non-real time environment. The

Abbreviations: BAC, blood alcohol concentration; NVDRS, National Violent Death Reporting System; SES, socioeconomic status. 
National Mortality Followback Survey, for instance, which was used by some of the mentioned studies, collected data from family and friends via telephone survey, but often six months or more after the original suicide event occurs. Medical record review, also retrospective, captures only data reported to the medical staff and for limited periods of treatment time. Medical examiner records alone may or may not capture the complete mental health and substance abuse history of a decedent, but will often contain toxicology data.

The National Violent Death Reporting System (NVDRS) attempts to resolve these types of timeliness, quality, and data consolidation issues. The NVDRS collects information from multiple sources at the time of the death and during the subsequent law enforcement and medicolegal death investigation, and consolidates the data into a unified repository. When combined with race/ethnicity variables, the NVDRS provides a rich database from which to assess the impact of race/ethnicity, substance abuse, and mental illness on suicide.

\section{METHODS}

The NVDRS is a state-based surveillance system that provides detailed accounts of violent deaths that occur in the US.$^{20}$ States are funded by the Centers for Disease Control and Prevention through a competitive grant system. Currently 17 states contribute data to the NVDRS with a goal of national coverage in the coming years. The NVDRS includes all suicides, homicides, legal intervention deaths, unintentional firearm deaths and deaths for which the manner is undetermined. ${ }^{21}$

The NVDRS is an incident-based system that collects data on all incidents and includes information on deceased victims within participating states, suspects, and weapons and the relationships between them. Victim variables include demographic data, date, time and place of injury and death, toxicology results, wound data, circumstances preceding the death, mental health and substance abuse diagnoses and treatment indicators, hospital International Classification of Disease, 10th revision (ICD-10) external cause of injury codes
(E-codes) if treated for the fatal injury, and detail on the weapon type. Suspect variables include demographics, relationship to the victim(s), and weapons used. Data on deaths that occur within 24 hours of one another and are linked to a single event are loaded as a single incident. Thus, a multiple suicide, or suicide pact, with deaths occurring in the same 24 hour period would be linked as one incident.

The NVDRS collects data from coroner/medical examiner records, police reports, death certificates, toxicology labs, crime labs, and Alcohol, Tobacco and Firearms gun trace reports. Individual states may have access to child fatality review teams and supplemental homicide reports that they may also include. Coroner/medical examiner and law enforcement reports often contain accounts from family and friends regarding mental illness and substance abuse history which can be supplemented and confirmed by toxicology findings. Very often, the information is collected at the time the victim is found by medicolegal death investigators and law enforcement, and often while persons who know the victim well are still at the death scene.

States manage data collection typically through state health departments or a subcontracted entity, such as a medical examiner's office. While there are over 700 variables in the NVDRS, some are duplicated for the expressed purpose of comparing the content of multiple data sources. For instance, a suicide victim's history of mental health treatment is entered twice; once from data provided by the coroner/medical examiner investigation and once by data provided from the police investigation. Both will likely have included family input as well as indications of treatment found on scene such as documentation of visits to mental health specialists, presence of psychotropic medications prescribed to the decedent or discharge papers from previous psychiatric admissions. Data are coded by an abstractor who is trained to extract data from the various types of reports, or data are imported from other systems (such as medical examiner data sets) and reviewed by the abstractor to ensure accuracy in the loading process.

\begin{tabular}{|c|c|c|c|c|}
\hline & $\begin{array}{l}\text { White (non- } \\
\text { Hispanic) \% }\end{array}$ & $\begin{array}{l}\text { Black (non- } \\
\text { Hispanic) \% }\end{array}$ & Hispanic \% & $\begin{array}{l}\text { Other (non- } \\
\text { Hispanic) \% }\end{array}$ \\
\hline Decedents tested & $n=5797$ & $n=501$ & $n=257$ & $n=310$ \\
\hline Alcohol & 74.9 & 89.8 & 76.3 & 72.6 \\
\hline Amphetamines & 38.1 & 40.5 & 45.5 & 33.9 \\
\hline Cocaine & 47.4 & 63.5 & 57.2 & 43.9 \\
\hline Opiates & 44.6 & 52.5 & 50.6 & 39.7 \\
\hline Marijuana & 28.2 & 29.1 & 39.3 & 22.6 \\
\hline Antidepressants & 38.2 & 36.7 & 41.6 & 32.9 \\
\hline \multicolumn{5}{|l|}{ Toxicology tests } \\
\hline Alcohol & $n=4342$ & $n=450$ & $n=196$ & $\mathrm{n}=225$ \\
\hline Present* & 34.3 & 25.6 & 40.3 & 28.4 \\
\hline Average BAC†‡ & 0.13 & 0.10 & 0.14 & 0.13 \\
\hline Amphetamines & $n=2211$ & $n=203$ & $n=117$ & $n=105$ \\
\hline Present & 4.3 & 0.5 & 5.1 & 3.8 \\
\hline Cocaine & $n=2748$ & $n=318$ & $n=147$ & $n=136$ \\
\hline Present* & 8.5 & 17.3 & 17.7 & 9.6 \\
\hline Opiates & $n=2587$ & $n=263$ & $n=130$ & $n=123$ \\
\hline Present* & 17.9 & 8.0 & 10.0 & 13.0 \\
\hline Marijuana & $n=1634$ & $n=146$ & $n=101$ & $n=70$ \\
\hline Present & 8.1 & 12.3 & 8.9 & 4.3 \\
\hline Antidepressants & $n=2215$ & $n=184$ & $n=107$ & $n=102$ \\
\hline Present ${ }^{*}$ & 26.8 & 14.7 & 10.3 & 16.7 \\
\hline Family/friends assessment & $n=5797$ & $n=501$ & $n=257$ & $n=310$ \\
\hline Alcohol problem ${ }^{*}$ & 17.5 & 8.6 & 19.1 & 17.4 \\
\hline Other substance abuse problem 1 & 14.0 & 14.6 & 17.1 & 10.3 \\
\hline
\end{tabular}


Table 2 Evidence of mental illness (MI) for suicide victims by race/ethnicity

\begin{tabular}{lllll} 
& $\begin{array}{c}\text { White (non- } \\
\text { Hispanic) \% }\end{array}$ & $\begin{array}{c}\text { Black (non- } \\
\text { Hispanic) \% }\end{array}$ & Hispanic \% & $\begin{array}{c}\text { Other (non- } \\
\text { Hispanic) \% }\end{array}$ \\
\hline Family/friend reporting and death scene & $\mathrm{n}=5797$ & $\mathrm{n}=501$ & $\mathrm{n}=257$ & $\mathrm{n}=310$ \\
evidence of diagnosis and treatment & & & & \\
Ever treated for mental illness* & 33.1 & 26.0 & 16.3 & 23.2 \\
Current mental illness* & 44.2 & 34.7 & 21.4 & 31.0 \\
Current treatment for MI* & 32.8 & 25.0 & 15.2 & 22.3 \\
History of suicide attempts* & 18.7 & 13.4 & 16.7 & 17.1 \\
Diagnosis of depression* & 34.7 & 21.6 & 15.6 & 24.2 \\
Diagnosis of bipolar disorder* & 6.1 & 2.6 & 2.3 & 2.3 \\
Diagnosis of schizophrenia* & 1.8 & 4.2 & 1.2 & 2.6 \\
Diagnosis of anxiety disorder & 2.6 & 1.6 & 0.8 & 1.6 \\
Diagnosis of PTSD & 0.6 & 0.8 & 0.0 & 0.7 \\
Diagnosis of ADD & 0.5 & 0.6 & 0.0 & 1.0 \\
Diagnosis of OCD & 0.3 & 0.2 & 0.0 & 0.0 \\
Diagnosis of eating disorder & 0.1 & 0.0 & 0.0 & 0.0 \\
Other diagnosis & 2.0 & 1.8 & 1.2 & 1.0 \\
Family/friends assessment & $\mathrm{n}=5797$ & $\mathrm{n}=501$ & $\mathrm{n}=257$ & $\mathrm{n}=310$ \\
Current depressed mood* & 46.8 & 30.7 & 45.1 & 43.9 \\
\hline * $\chi^{2}$ tests of differences significant at O.05 or less. & & & \\
PTSD, post-traumatic stress disorder; ADD, attention deficit disorder; OCD, obsessive compulsive disorder. & \\
\hline
\end{tabular}

Suicide incidents were identified for calendar year 2004 by the assigned manner of death variable and the presence of any code in the race/ethnicity variables. The 2004 data set includes suicides from 13 states: Alaska, Colorado, Georgia, Maryland, Massachusetts, New Jersey, North Carolina, Oklahoma, Oregon, Rhode Island, South Carolina, Virginia, and Wisconsin. Medical examiner and law enforcement investigator findings, including results of interviews with family and friends, are included for each of these cases as they pertain to mental health and substance abuse history. Toxicology results are presented for all cases when available. Toxicology data are not complete for all cases; each coroner/ medical examiner has the authority to test, or not test, for alcohol and individual types of drugs as they deem appropriate. The number of cases for which toxicology tests were ordered and returned as "positive" is presented below. Specific blood alcohol concentration (BAC) levels are also provided.

Tests of significance across racial/ethnic groups were calculated using a null hypothesis of no difference between groups and $\chi^{2}$ tests of difference. Due to marked nonnormality of data, the non-parametric Kruskal-Wallis test was used for assessing the differences among racial/ethnic groups in BAC.

\section{RESULTS}

Selection of NVDRS suicide incidents resulted in 6913 victims from the 13 participating states during calendar year 2004. Forty eight of those victims were missing data on race/ ethnicity $(<0.01 \%)$ and were omitted from the study. Of the 6865 included victims, 5797 (84.4\%) were non-Hispanic white, 501 (7.3\%) were non-Hispanic black, 257 (3.7\%) were Hispanic, and $310(4.5 \%)$ were from other racial/ethnic groups.

\section{Toxicology}

Differences exist among the racial/ethnic groups in the percentage of decedents tested for alcohol and drugs at the time of autopsy. Non-Hispanic blacks were tested more often than other racial/ethnic groups for alcohol, cocaine, and opiates while Hispanics were tested more often than other racial/ethnic groups for amphetamines and marijuana. Significant differences were found in the presence of alcohol, cocaine, opiates, and antidepressants among the racial/ethnic groups. A detailed comparison of toxicology results is shown in table 1 .
A test for BAC was requested by coroners/medical examiners in $74.9 \%$ of non-Hispanic white decedents, $89.8 \%$ of nonHispanic blacks, $76.3 \%$ of Hispanics, and $72.6 \%$ of decedents of other races/ethnicities. Of those tested, $34.3 \%$ of non-Hispanic whites, $25.6 \%$ of non-Hispanic blacks, $40.3 \%$ of Hispanics, and $28.4 \%$ from other racial/ethnic groups tested positive for alcohol at the time of death $\left(\chi^{2}=20.6, \mathrm{df}=3, \mathrm{p}<0.01\right)$. The mean BAC at the time of death was $0.13 \mathrm{~g} / \mathrm{dl}$ for non-Hispanic whites, $0.10 \mathrm{~g} / \mathrm{dl}$ for non-Hispanic blacks, $0.14 \mathrm{~g} / \mathrm{dl}$ for Hispanics, and $0.13 \mathrm{~g} / \mathrm{dl}$ for other racial/ethnic groups (Kruskal-Wallis $\mathrm{p}=0.02$ ). Thus, a lower proportion of nonHispanics blacks had evidence of alcohol at the time of death among those who were tested and lower blood alcohol concentrations compared with other groups.

A test for cocaine was completed for $47.4 \%$ of non-Hispanic white decedents, $63.5 \%$ of non-Hispanic blacks, $57.2 \%$ of Hispanics and $43.9 \%$ of decedents from other racial/ethnic groups. Among those tested, a lower proportion of nonHispanic whites tested positive for cocaine (8.5\% of cases) when compared to non-Hispanic blacks (17.3\%), Hispanics $(17.7 \%)$, and decedents of other groups $(9.6 \%)\left(\chi^{2}=36.1\right.$, $\mathrm{df}=3, \mathrm{p}<0.01)$.

Opiate testing was completed for $44.6 \%$ of non-Hispanic white decedents, $52.5 \%$ of non-Hispanic blacks, $50.6 \%$ of Hispanics, and $39.7 \%$ of decedents from other racial/ethnic groups. Among those tested, a higher proportion of nonHispanic whites tested positive for opiates (17.9\%) when compared to non-Hispanic blacks (8.0\%), Hispanics (10.0\%), and non-Hispanics from other racial/ethnic groups $(13.0 \%)$ $\left(\chi^{2}=22.5, \mathrm{df}=3, \mathrm{p}<0.01\right)$. A similar pattern was found among those for whom the presence of antidepressants was evaluated. Antidepressants were evaluated in $38.2 \%$ of nonHispanic white decedents, $36.7 \%$ of non-Hispanic blacks, $41.6 \%$ of Hispanics, and $32.9 \%$ of decedents of other races/ ethnicities. Of those tested, a higher proportion of nonHispanic whites tested positive for antidepressants $(26.8 \%)$ when compared to non-Hispanic blacks (14.7\%), Hispanics $(10.3 \%)$, and non-Hispanics from other racial/ethnic groups $(16.7 \%) \quad\left(\chi^{2}=30.4, \mathrm{df}=3, \mathrm{p}<0.01\right)$. Thus, fewer nonHispanic whites had evidence of cocaine in their system at the time of death, but more non-Hispanic whites compared with those tested in other groups had evidence of opiates and antidepressants in their drug screens.

Family members and friends who were interviewed during the investigation process reported significantly different rates of alcohol problems among the decedents based on their own 


\begin{tabular}{|c|c|c|c|}
\hline & 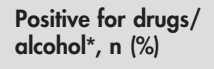 & 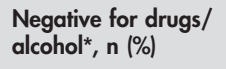 & Total \\
\hline \multicolumn{4}{|c|}{ Controlling for non-Hispanic white $†$} \\
\hline Mental health problem & $770(17.4)$ & $1000(22.5)$ & 1770 (39.9) \\
\hline No mental health problem & $1255(28.3)$ & $1409(31.8)$ & $2664(60.1)$ \\
\hline Total & $2025(45.7)$ & 2409 (54.3) & $4434(100)$ \\
\hline \multicolumn{4}{|c|}{ Controlling for non-Hispanic black $†$} \\
\hline Mental health problem & $36(7.8)$ & 88 (19.2) & $124(27.0)$ \\
\hline No mental health problem & $133(29.0)$ & $202(44.0)$ & $335(73.0)$ \\
\hline Total & $169(36.8)$ & $290(63.2)$ & $459(100)$ \\
\hline \multicolumn{4}{|l|}{ Controlling for Hispanic } \\
\hline Mental health problem & $18(9.0)$ & $19(9.6)$ & $37(18.6)$ \\
\hline No mental health problem & $80(40.2)$ & $82(41.2)$ & $162(81.4)$ \\
\hline Total & $98(49.2)$ & $101(50.8)$ & $199(100)$ \\
\hline \multicolumn{4}{|c|}{ Controlling for non-Hispanic other races } \\
\hline Mental health problem & 25 (10.9) & $41(17.8)$ & $66(28.7)$ \\
\hline No mental health problem & $61(26.5)$ & $103(44.8)$ & $164(71.3)$ \\
\hline Total & $86(37.4)$ & $144(62.6)$ & $230(100)$ \\
\hline
\end{tabular}

perceptions. Family and friends mentioned an alcohol problem in $17.5 \%$ of non-Hispanic white decedents, $8.6 \%$ of non-Hispanic blacks, $19.1 \%$ of Hispanics, and in $17.4 \%$ of cases for non-Hispanics of other races/ethnicities $\left(\chi^{2}=27.0\right.$, $\mathrm{df}=3, \mathrm{p}<0.01)$. Family reported data for non-Hispanic blacks were consistent with toxicology data in that families and friends of non-Hispanic blacks reported fewer alcohol problems and the toxicology data showed less alcohol at the time of death. For all other racial/ethnic groups, family and friends consistently reported that $17 \%-19 \%$ of decedents had alcohol problems but nearly double that percentage tested positive at the time of the suicide. No significant differences were found in the presence of amphetamines, marijuana, or family and friends' evaluation of a drug problem.

\section{Mental illness}

Significant differences were found among racial/ethnic groups in type of mental illness diagnosed and the receipt of current and past treatment. Based on family and friend reports and scene evidence, Hispanics were less likely to have been professionally diagnosed with a mental illness $\left(\chi^{2}=82.4\right.$, $\mathrm{df}=3, \mathrm{p}<0.01)$ or treated for one either currently $\left(\chi^{2}=58.2\right.$, $\mathrm{df}=3, \mathrm{p}<0.01)$ or ever in their lives $\left(\chi^{2}=50.9, \mathrm{df}=3, \mathrm{p}<0.01\right)$. However, family and friends report just as much perceived depression for Hispanics (45.1\%) as for all other racial/ethnic groups combined $(43.7 \%)$. A detailed comparison of mental illness results as reported by family and friends, or based on evidence at the scene, appears in table 2.

More non-Hispanic whites had evidence of depression $\left(\chi^{2}=83.5, \mathrm{df}=3, \mathrm{p}<0.01\right)$ or bipolar disorder $\left(\chi^{2}=23.5\right.$, $\mathrm{df}=3, \mathrm{p}<0.01)$ at the time of death compared with other groups. This is consistent with the data in table 1, indicating that more non-Hispanic whites had evidence of antidepressant and opiate use at the time of death than all other groups. More non-Hispanic blacks had evidence of schizophrenia $\left(\chi^{2}=15.4, \mathrm{df}=3, \mathrm{p}<0.01\right)$ and fewer had attempted suicide before the completed event $\left(\chi^{2}=9.3, \mathrm{df}=3, \mathrm{p}=0.03\right)$. NonHispanic blacks were also reported by their family and friends to have been less depressed based on their own perceptions than all other racial/ethnic groups included in this study $\left(\chi^{2}=8.2, \mathrm{df}=3, \mathrm{p}<0.01\right)$.

No significant differences were found for the other diagnoses including anxiety disorders, post-traumatic stress disorder, attention deficit disorder, obsessive compulsive disorder, eating disorder, or other disorders.

\section{Substance abuse and mental illness}

Non-Hispanic whites $\left(\chi^{2}=5.6, \mathrm{df}=1, \mathrm{p}=0.02\right)$ and nonHispanic blacks $\left(\chi^{2}=4.4, \mathrm{df}=1, \mathrm{p}=0.04\right)$ were more likely to have substance abuse and mental health problems as comorbid conditions than Hispanics $\left(\chi^{2}=0.01, \mathrm{df}=1\right.$, $\mathrm{p}=0.94)$ and non-Hispanics of other races/ethnicities $\left(\chi^{2}=0.01, \mathrm{df}=1, \mathrm{p}=0.92\right)$. The conditional probability of having a mental health problem given a substance abuse problem was 0.38 for non-Hispanic whites, and 0.44 for having a substance abuse problem given a mental health problem. For non-Hispanic blacks the conditional probability of having a mental health problem given a substance abuse problem was 0.21 and 0.29 for a substance abuse problem given a mental health problem. Thus, the conditional probability of having one condition given the other was higher for non-Hispanic whites than non-Hispanic blacks.

Although the conditional probabilities of one condition given the other are close for all other racial/ethnic groups, this is not true for Hispanics. The conditional probability of having a mental health problem given a substance abuse problem for Hispanics was 0.18 and 0.48 for a substance abuse problem given a mental health problem. Thus, Hispanics were more likely to have a substance abuse problem, but no mental health problem. This finding is consistent with the previous findings that fewer Hispanics had evidence of mental illness, despite family reported depression levels that are similar to other racial/ethic groups. A stratified analysis of race/ethnicity, substance abuse, and mental illness for suicide victims is presented in table 3.

\section{DISCUSSION}

The NVDRS 2004 data on the impact of race/ethnicity, substance abuse, and mental illness as they relate to completed suicide identify a few findings that might be incorporated into prevention and treatment programs. Specifically, Hispanic families reported perceived levels of depression in decedents similar to the perceived levels of depression in other racial/ethnic groups. However, there was less evidence of current and lifetime treatment, sometimes as much as half the treatment levels reported for other groups. 
Hispanics were also less likely to experience substance abuse problems and mental health problems as comorbid illnesses, instead experiencing substance abuse problems in the absence of other mental health problems. This might be due to lack of access to mental healthcare due to socioeconomic status (SES), or perhaps to potential stigma in the Hispanic community regarding mental illness and treatment. Providing expanded access to community mental health clinics/practitioners and working in the Hispanic community to reduce the social stigma associated with mental illness might increase diagnosis and treatment rates. Also, it is imperative that practitioners listen to family members and friends who report significant depression with another family member, even if that family member denies it.

Unlike Hispanics, more non-Hispanic whites had evidence of a formal treatment diagnosis, to have been under a doctor's care, and taking antidepressants and/or opiates at the time of death. However, although nearly $45 \%$ of the suicide victim's families in this group reported a current, professionally diagnosed mental illness, only $32.8 \%$ were currently being treated. A similar pattern was seen among the other racial/ethnic groups. Working to increase access to treatment and reduce the stigma associated with it might encourage ongoing mental health treatment among those who tend to drop out of treatment. Non-Hispanic whites were also more likely to have comorbid mental health and substance abuse issues, suggesting the need for programs that treat both issues concurrently.

Data for non-Hispanic blacks suggest unique prevention and treatment strategies might be appropriate as there is less evidence of depression, alcohol abuse, prior suicide attempts, and comorbid mental illness and substance abuse, all typical screening indicators for suicide. Non-Hispanic blacks might be at increased risk if there is a diagnosis of schizophrenia or a history of cocaine use, not necessarily as comorbid conditions but more likely as individual illnesses.

The findings in this paper are consistent with much of the current literature about the prevalence of substance abuse and mental illness as it relates to race/ethnicity and completed suicide; depression was the most prevalent mental illness, ${ }^{16-19}$ there was evidence of high levels of alcohol or other drugs used at the time of death, ${ }^{12}{ }^{12-15}$ alcohol levels were often higher than the legal intoxication levels in most states, ${ }^{11}$ and comorbid mental illness and substance abuse were common. There was also evidence, however, that unique patterns exist among racial/ethnic groups to suggest that different suicide prevention and treatment strategies and indicators might be appropriate.

There are limitations to the data, particularly with respect to the completion and interpretation of toxicology tests, validation of mental illness diagnoses and treatment, identification of suicide cases, and the lack of data on SES in the NVDRS which prevents us from evaluating how much of our observed racial/ethnic differences might be due to SES.

Additional research is needed to understand how specific differences in the administration of toxicology tests might be impacting these results. Testing of non-Hispanic black and Hispanic decedents at higher rates than other groups might be reflective of societal bias around suspicion of drug use in these racial/ethnic groups. Among all racial/ethnic groups, approximately $10-30 \%$ of cases did not receive any substance intoxication tests. There is also the possibility that some decedents might have tested positive for alcohol as a result of the body decomposition process which, over time, produces small but detectible amounts of alcohol in the body. Testing of every death by suicide, and the elimination of cases where a very low positive alcohol content might be the result of decomposition, might produce higher or lower rates of evidence of alcohol or drug use at the time of death. In

\section{Key points}

- At the time of the suicide event, non-Hispanic blacks had lower blood alcohol contents, non-Hispanic whites more antidepressants and opiates and no differences were found in levels of amphetamines or marijuana by race and ethnicity.

- Hispanics were less likely to have been diagnosed with a mental illness or to have received treatment, although family reports of depression were comparable to nonHispanic whites and other racial and ethnic groups.

- Non-Hispanic whites were more likely to be diagnosed with depression or bipolar disorder and non-Hispanic blacks with schizophrenia.

- Analysis of comorbid substance abuse and mental health problems by race shows that non-Hispanic whites and non-Hispanic blacks are more likely to have comorbid substance abuse and mental health problems; Hispanics are more likely to have a substance abuse problem without comorbid mental health problems.

- The data suggest that suicide prevention efforts must address not only substance abuse and mental health problems in general, but the unique personal, family, and social characteristics of different racial and ethnic groups.

addition, this study does not control for mechanism of death. If suicide rates by medication overdose differ among racial/ ethnic groups, the drug abuse percentages might be inflated, especially for opiates. In these cases the positive test for opiates might reflect only the suicide act, or prescribed therapeutic doses and not a chronic drug problem. Despite these limitations, the toxicology results found in this study are consistent with the ranges of alcohol and drug levels found in the studies previously cited.

In addition, further validation of mental illness diagnoses and treatment, beyond family report and evidence on scene of mental health appointments, hospital admissions, or psychotropic medications might result in higher rates as the number of informants increases. While the mental health variables are derived from a compilation of all data sources in the NVDRS, cases for which informants report no mental illness and no treatment to all investigating agencies will be coded in that manner in the absence of contrary evidence at the death scene. If social stigma regarding the acknowledgement of mental illness or substance abuse is impacting family and friend's willingness to report problems, rates in this study might be underreported. A lack of longitudinal detail regarding acknowledged substance abuse and mental health disorders also does not allow us to examine the temporal order of these illnesses for these victims.

Finally, although the NVDRS attempts to capture all suicides by initiating cases and collecting data from multiple sources, it is still possible that some incidents were not identified. This occurs when, in the absence of absolute evidence of suicidal intent (that is, suicide note) or under pressure from families or other interested parties, some suicides might be assigned the manner of death of "undetermined" or "unintentional" rather than suicide. The NVDRS does collect data for all "undetermined" cases, and thus a comparative study of these cases and confirmed suicides is possible. The NVDRS does not collect data on "unintentional" cases (except unintentional firearm deaths), as these are considered accidental and not the result of 
suicidal violence. A recent study showed the variation among NVDRS states in the rates of cases classified as "undetermined" to be between $1.8 \%$ and $60.0 \%$ in 2003 and between $0.4 \%$ and $50.1 \%$ in $2004 .{ }^{22}$ Age adjusted "unintentional" deaths from the National Vital Statistics System for NVDRS states for 1999-2003 ranged from $0.86 / 100000$ residents to $9.87 / 100000$ residents. $^{22}$ A portion of these cases might actually be suicides.

Mental illness and substance abuse play a significant role in contributing to suicide events and racial/ethnic differences are noted across many suicide circumstances. Numerous other factors might be involved as well. The NVDRS is the only data system that regularly collects and consolidates information from multiple sources on all violent deaths occurring in participating states. In addition to race/ethnicity, substance abuse, and mental illness, the NVDRS has the capability, through hundreds of other variables and numerous ongoing studies, to greatly enhance our understanding of the causes and circumstances preceding suicide and how to improve risk factor identification and treatment of potential victims.

\section{Authors' affiliations}

D L Karch, L Barker, T W Strine, Centers for Disease Control and Prevention, National Center for Injury Prevention and Control, Atlanta, GA, USA

Competing interests: none.

The findings and conclusions in this paper are those of the authors and do not necessarily represent the views of the Centers for Disease Control and Prevention.

\section{REFERENCES}

1 Centers for Disease Control and Prevention, National Center for Injury Prevention and Control. Web-based Injury Statistics Query and Reporting System (WISQARS). Available at http://www.cdc.gov/ncipc/wisqars (accessed March 2006)

2 Cottler LB, Campbell W, Krishna VA, et al. Predictors of High Rates of Suicidal Ideation Among Drug Users. J Nerv Ment Dis 2005;193:431-7.

3 Havens JR, Ompad DC, Latkin CA, et al. Suicidal ideation among AfricanAmerican non-injection drug users. Ethn Dis 2005;15:110-15.
4 Friedman AS, Terras A, Zhu W, et al. Depression, negative self-image, and suicidal attempts as effects of substance use and substance dependence. $J$ Addict Dis 2004;23:55-71.

5 Kaslow NJ, Price AW, Wyckoff S, et al. Person factors associated with suicidal behavior among African-American women and men. Cultur Divers Ethnic Minor Psychol 2004;10:5-22.

6 Roy A. African American and Caucasian attempters compared for suicide risk factors: a preliminary study. Suicide Life Threat Behav 2003;33:443-7.

7 Kelly TM, Cornelius JR, Lynch KG. Psychiatric and substance use disorders as risk factors for attempted suicide among adolescents: a case control study. Suicide Life Threat Behav 2002;32:301-12.

8 Brent DA. The psychological autopsy: methodological considerations for the study of adolescent suicide. Suicide Life Threat Behav 1989;19:43-57.

9 Havasi B, Magori K, Toth A, et al. Fatal suicide cases from 1991-2000 in Szeged, Hungary. Forensic Sci Int 2005; 147(Suppl):S25-8.

10 May PA, Van Winkle NW, Williams MB, et al. Alcohol and suicide death among American Indians of New Mexico: 1980-1998. Suicide Life Threat Behav 2002;32:240-55.

11 Crombie IK, Pounder DJ, Dick PH. Who takes alcohol prior to suicide? J Clin Forensic Med 1998;5:65-8.

12 Garlow SJ. Age, gender, and ethnicity differences in patterns of cocaine and ethanol use preceding suicide. Am J Psychiatry 2002;159:615-19.

13 Hunt IM, Robinson J, Bickley $\mathrm{H}$, et al. Suicides in ethnic minorities within 12 months of contact with mental health services: national clinical survey. Br J Psychiatry 2003;183:155-60.

14 Castle K, Duberstein PR, Meldrum S, et al. Risk factors for suicide in blacks and whites: an analysis of data from the 1993 National Mortality Feedback Survey. Am J Psychiatry 2004;161:395-7.

15 Marzuk PM, Tardiff K, Leon AC, et al. Prevalence of cocaine use among residents of New York City who committed suicide during a one-year period. Am J Psychiatry 1992;149:371-5.

16 Bertolote JM, Fleischmann A, De Leo D, et al. Psychiatric diagnoses and suicide: revisiting the evidence. Crisis 2004;25:147-55.

17 Perenyi A, Forlano R. Suicide in schizophrenia. Neruopsychopharmocol Hungarica 2005;7:107-17

18 Kung HC, Pearson JL, Liu X. Risk factors for male and female suicide decedents age 14-64 in the United States. Results from the 1993 National Mortality Followback Survey. Soc Psychiatry Psychiatr Epidemiol 2003;38:419-26

19 Kung HC, Liu X, Juon HS. Risk factors for suicide in Caucasians and in African-Americans: a matched case-control study. Soc Psychiatry Psychiatr Epidemiol 1998;33:155-61

20 Azreal D, Barber C, Mercy J. Linking data to save lives: recent progress in establishing a National Violent Death Reporting System. Harvard Health Policy Review $2001 ; 2: 38-42$.

21 Paulozzi LJ, Mercy J, Frazier Jr L, et al. CDC's National Violent Death Reporting System: background and methodology. Inj Prev 2004;10:47-52.

22 Breiding MJ, Wiersema B. Variability of undetermined manner of death classification in the US. Inj Prev 2006;12(Suppl II):ii49-ii54. 